\section{COPD: Inhalative Steroide erhöhen Risiko für Mund-Soor dosisabhängig}

Dekhuijzen PNR et al. Incidence of oral thrush in patients with COPD prescribed inhaled corticosteroids: Effect of drug, dose, and device. Respir Med 2016; 120: $54-63$

Mund-Soor ist eine bekannte Nebenwirkung inhalativer Steroide bei Patienten mit Asthma. Obwohl auch Patienten mit einer COPD sehr oft inhalative Steroide verschrieben bekommen, gibt es bei ihnen nur recht wenige Daten zur Frage, wie häufig Mund-Soor bei ihnen auftritt. Eine Studie ging nun dieser Frage nach und untersuchte auch, ob sich hier gegebenenfalls eine Abhängigkeit von Dosis, Art des Wirkstoffs und Applikationsform zeigt.

Die Autoren führten hierzu eine retrospektive Kohortenstudie durch, für die sie Einträge einer medizinischen Datenbank mit mehr als 2,4 Millionen Patienten aus über 550 Hausarztpraxen nutzten. Eingang in die Analyse fanden Patienten mit einer COPD, die zum Zeitpunkt des Einschlusses 40 Jahre und älter waren, über einen mindestens 2-jährigen dokumentierten Krankheitsverlauf verfügten und zwei oder mehr Verordnungen von Fix-Dosis-Kombinationen aus inhalativem Steroid (ICS) und langwirksamem Bronchodilatator (LABA) oder einem alleinigen LABA in der Medikation hatten. Primärer Endpunkt war die Inzidenz eines Mund-Soors. Die Autoren verglichen hierbei verschiedene Kombinationstherapien (Budesonid/Formoterol und Fluticason/Salmeterol) und Applikationsformen (Trockenpulver-Inhalator und treibgasbetriebene Dosieraerosole).

In die Analyse gingen jeweils $8255 \mathrm{~Pa}$ tienten ein, die entweder eine Kombination aus ICS und LABA oder nur einen LABA erhielten. Das Durchschnittsalter der Patienten lag bei 69 Jahren, 57\% waren Männer, die Mehrheit hatte früher geraucht (50\%) oder rauchte noch
(40\%). Patienten, die eine Kombination aus ICS und LABA erhielten, hatten signifikant häufiger einen Mund-Soor als Patienten mit alleiniger LABA-Medikation (5,5\% vs. $2,7 \%$, Odds Ratio 2,18). Allerdings trat der Soor unter der Kombination aus Budesonid und Formoterol signifikant seltener auf als unter der Kombination aus Fluticason und Salmeterol (5,7\% vs. 7,0\%, Odds Ratio 0,77). Ähnliches galt auch unter Medikation mit Fluticason und Salmeterol im Hinblick auf die Applikationsform (treibgasbetriebene Dosieraerosole 5,5\% vs. Trockenpulver-Inhalator 7,3\%, Odds Ratio $0,67)$. Auch bezüglich der Dosis zeigte sich unter Fluticason und Salmeterol eine Assoziation mit dem Soor-Risiko (Odds Ratio 1,97 für hohe $[\geq 1000 \mu \mathrm{g} / \mathrm{d}$; $7,2 \%$ vs. niedrige $[<500 \mu \mathrm{g} / \mathrm{d} ; 4,7 \%$ ] Dosis), im Falle der Kombination Budesonid/Formoterol fand sich bei ähnlichem Trend (7,4\% vs. 5,6\%) kein signifikanter Unterschied.

\section{FAZIT}

Der Gebrauch inhalativer Steroide erhöhte bei Patienten mit COPD das Risiko für einen Mund-Soor, wobei sich für die Kombination aus Fluticason und Salmeterol eine Dosisabhängigkeit zeigte. Einen protektiven Effekt zeigen möglicherweise ein treibgasbetriebenes Dosieraerosol für diese Kombination oder Budesonid/Formoterol als Trockenpulver-Inhalator, so die Autoren.

Dr. med. Johannes Weiß, Bad Kissingen 